Abstract PS1-20

Fiscal Tracking of Clinical Research: Marshfield Clinic Research

Foundation Community Clinical Oncology Program

Kim M. Fuehrer, RN, BSN, MSM, Marshfield Clinic Research Foundation; Tarit K. Banerjee, MD, FACP, Marshfield Clinic

Background: Marshfield Clinic Research Foundation (MCRF) is the research division of Marshfield Clinic. MCRF has received 25 years of Community Clinical Oncology Program (CCOP) funding from the National Cancer Institute (NCI). Grant funds require that each CCOP attain a specified number of credits through new participant enrollment and follow-up each year. The Principal Investigator (PI) and CCOP Administrator are responsible for tracking annual CCOP revenues and expenditures as well as CCOP credits. Revenue and expense details involved in the performance of CCOP clinical trials are difficult to track. Limited information is available regarding the true cost of CCOP clinical trials. Methods: Marshfield Clinic Research Foundation (MCRF) developed a tracking system utilizing electronic and manual processes, and established individual accounts for each research base within each source of revenue such as the NCI CCOP grant, pass-through additional federal and industry funds, additional private funds. Within each individual account established, expenditures were tracked electronically by various cost categories such as staff salaries and benefits (based on time and effort reports), laboratory sample preparation for send-outs, travel expenses to attend research-base meetings, copying, printing, telephone. Results: This poster provides general information and graphic depictions of how MCRF tracked CCOP revenues and expenditures from June 1, 2006 through May 31, 2007. Credit information per research-base and total by treatment, cancer control and follow-up credit is also provided. The data indicated that $52 \%$ of the expenses were funded by NCI grant funding, $12 \%$ from additional funds provided by the research bases, $29 \%$ from institutional support, and $7 \%$ from other funds donated for oncology research. Conclusions: To sustain a CCOP, significant financial support by the recipient institution is essential over and above the support of the infrastructure. During 2006-2007, MCRF recorded $\$ 4600$ in expenses for each goal credit.

Abstract PS1-21

Radiotherapy Delay in Older Women With Early-Stage Breast Cancer

Heather Taffet Gold, PhD, Weill Cornell Medical College; Soe Soe Thwin, PhD, Boston University; Diana S.M. Buist, PhD, MPH, Group Health Center for Health Studies; Terry Field, DSc, Meyers Primary Care Institute; Rebecca A. Silliman, MD, $\mathrm{PhD}$, Boston University

Objective: To assess predictors of delayed radiotherapy (RT) and the potential impact of RT delay on breast cancer outcomes in older women with earlystage breast cancer. Methods: We studied 616 women, ages 65 years or older, who were diagnosed with early-stage breast cancer from 1990-1994 at six HMOs. These women were a subset of the Cancer Research Network's Breast Cancer Treatment Effectiveness in Older Women (BOW) cohort. All women included underwent breast conserving surgery and radiotherapy, but no chemotherapy. We examined whether race, age, estrogen receptor (ER) or progesterone receptor (PR) status, Charlson comorbidity index at diagnosis, tamoxifen use, tumor size, histology, and lymph-node positivity were associated with RT delays of more than 8 weeks post-surgery using chi-square tests and multivariate logistic regression. We applied Cox proportional hazards models to assess whether RT delay and demographic and clinical variables were associated with 10-year breast cancer recurrence rates. Results: Of the 82 women with delayed RT, 17 (21\%) recurred, whereas only 73 of $534(14 \%)$ without delay recurred $(P=0.09)$. Of those with delays $>8$ weeks, mean delay was 114 days (16 weeks) and median was 69.5 days (10 weeks). African American race compared to White race was associated with delayed RT (OR, $3.1 ; 95 \%$ CI, 1.5-6.6) in multivariate analyses that controlled for demographic and clinical variables and HMO site. Breast cancer tumors larger than $2 \mathrm{~cm}$ were associated significantly with higher recurrence rates $(\mathrm{HR}=4.2 ; 95 \% \mathrm{CI}$, $2.2-8.1$ ), as were subjects with more than 4 positive lymph nodes $(\mathrm{HR}=6.6$; $95 \%$ CI, 1.9-22.7). There was a trend towards higher recurrence rates for subjects with RT delay (HR=1.7; 95\% CI, 0.91-3.0). Conclusions: Although African American race was associated with RT delay, RT delay itself was not strongly associated with recurrence rates. Tumor characteristics are the major predictors of recurrence in this population of breast cancer patients.
Abstract PS1-22

Differences in Treatment of Ductal Carcinoma In Situ by Race/ Ethnicity in Large Integrated Health Plans

Reina Haque, PhD, MPH, Kaiser Permanente Southern California; Ninah Achacoso, PhD, MPH, Kaiser Permanente Northern California; Suzanne W. Fletcher, MD, MSc, Harvard Medical School; Larissa Nekhlyudov, MD, Harvard Pilgrim Health Care; Laura Collins, MD, Beth Israel Deaconess Medical Center; Stuart J. Schnitt, MD, Beth Israel Deaconess Medical Center; Charles P. Quesenberry, PhD, Kaiser Permanente Northern California; Michelle M. McGuire, MA, Kaiser Permanente Southern California; Laurel A. Habel, PhD, Kaiser Permanente Northern California

Background and Aims: The diagnosis of ductal carcinoma in situ (DCIS) has increased markedly in the U.S. from 4,800 in 1983 to 59,000 in 2005. Although racial differences exist in the detection, diagnosis, evaluation, and treatment for invasive breast cancer, the extent of disparity for DCIS is unclear. Because women with DCIS are at high risk for local recurrence or invasive disease, it is critical to determine the degree of treatment disparities among racial/ethnic groups. We examined racial/ethnic variation in adjuvant therapy in a cohort of 3,000 women diagnosed with DCIS between 1990 and 2001 and treated with breast conserving surgery in three geographically diverse health plans based in California and Massachusetts. Methods: We reviewed medical records and abstracted information regarding DCIS treatment, demographic characteristics, and comorbidity. Stratified analyses and multinomial logistic regression were conducted. Main Findings: After adjusting for age, diagnosis year, study site, and geocoded income, odds ratios suggest that minority women were as likely to undergo adjuvant radiation therapy as white women. Asian/Pacific Islander women were somewhat more likely to receive adjuvant tamoxifen therapy (adjusted OR, $1.3 ; 95 \% \mathrm{CI}, 0.7-2.2$ ) compared to white women; however, there was little difference between white women and Hispanic women (adjusted OR, 0.8; 95\% CI, 0.4-1.7) or black women (adjusted OR, 0.9; 95\% CI, 0.5-1.7). Black women were somewhat less likely than white women to undergo a combination of adjuvant tamoxifen and radiation therapy (adjusted OR, 0.6; 95\% CI, 0.4-1.0). Combination therapy was used to a similar extent in whites, Hispanics and Asian/Pacific Islanders. In a subset of patients for whom we had pathology data, tumor size and histology were controlled for in the analysis of adjuvant radiation therapy; however, these results were generally similar to those of the whole cohort. Conclusion: We found modest, though statistically insignificant, racial/ethnic differences in DCIS treatment. The similar use of adjuvant therapy by Asian, Hispanic, and white women is impressive and may be due to the general access to care in these managed care plans. The reasons for possible lower use of combined therapy among black women are unclear and require further study.

\section{Abstract PS1-23 \\ Control of Bowel Function by Colorectal Cancer Survivors With Permanent Intestinal Stomas: Behavioral Strategies}

Mark C. Hornbrook, PhD, Center for Health Research, Kaiser Permanente Northwest; Marcia Grant, PhD, RN, City of Hope National Medical Center; Jane Mohler, PhD, University of Arizona; Christopher Wendel, MS, Southern Arizona VA Health Care System; Carmit McMullen, PhD, Kaiser Permanente Northwest; Stephen J. Coons, PhD, University of Arizona; Carol M. Baldwin, $\mathrm{PhD}, \mathrm{RN}$, University of Arizona; Sylvan B. Green, MD, Arizona State University; Lisa Herrinton, $\mathrm{PhD}$, Kaiser Permanente Northern California; Robert S. Krouse, MD, Southern Arizona VA Health Care System

Background: There are a growing number of colorectal cancer (CRC) survivors in the United States, many of whom live with a permanent intestinal stoma (ostomy). Bowel function control has tremendous implications to overall health-related quality of life (HR-QOL). Methods: Long-term ( $>5$ years) CRC survivors with and without ostomies from three KaiserPermanente sites (Northwest, Northern California, Hawaii) were surveyed utilizing the City of Hope Quality of Life (mCOH-QOL)-Ostomy, followed by focus groups for patients with higher and lower HR-QOL. We compared HR-QOL for items specific for dietary changes and bowel function. Qualitative research techniques were used to analyze the open-ended questions, with the investigation team coding entries independently, reviewing discrepant codes, and reaching coding consensus. Results: Two hundred eighty-six ostomate and 395 non-ostomate CRC 
survivors completed the survey. While more controls felt comfortable with their diet less than one month after operation ( $48.6 \%$ vs $41.3 \%)$, a greater proportion took over one year or never felt comfortable with their diet $(21.9 \%$ vs $18.0 \%)$. Ostomates had less problems with constipation $(P=0.001)$, and more with gas $(P=0.02)$ than controls. More cases avoided vegetables $(P=0.007)$, while a greater proportion of controls avoided carbonated beverages $(P<0.001)$ and utilized specialty diets $(P=0.03)$. There were multiple dietary and behavioral changes utilized to control bowel habits, including the timing of eating, exercise, and food avoidance. Conclusions: CRC survivors utilize multiple techniques to control their bowel function, and thus control their lives. Insight into these methods can lead to meaningful interventions for this population of cancer survivors.

\section{Abstract PS1-24 \\ Fatigue and Sleep Quality of Persons with Ostomies: \\ Gender Differences}

Mark C. Hornbrook, PhD, Center for Health Research, Kaiser Permanente Northwest; Carol M. Baldwin, PhD, RN, Arizona State University; Christopher Wendel, MS, Southern Arizona VA Health Care System; Marcia Grant, PhD, RN, City of Hope Medical Center; Lisa Herrinton, PhD, Kaiser Permanente Northern California; Jane Mohler, PhD, Arizona State University; Carmit McMullen, PhD, Kaiser Permanente Northwest; Robert S. Krouse, MD, Southern Arizona VA Health Care System

Background: Some colorectal cancer (CRC) patients require a permanent ostomy (a surgically created opening on the exterior of the abdomen that allows feces to be eliminated from the body). An ostomy can create psychosocial distress and affect body image, sense of self, and sexual functioning. Few studies have examined health-related quality of life (HR-QOL) relevant to disturbed sleep of CRC survivors with ostomies; moreover, no studies have examined gender differences of ostomates on quality of sleep and fatigue. Methods: HR-QOL was examined in this cross-sectional study of long-term ( $>5$ years) CRC survivors with ostomies, who receive care at Kaiser Permanente health systems in California, Oregon, Washington, and Hawaii. Participants completed the City of Hope Quality of Life Ostomy questionnaire, which includes demographic and scaled items, and narrative comments for greatest challenges associated with having an ostomy. Two items, 'fatigue' and 'sleep disruption' (scale from 0 to 10), served as dependent variables. Age, ethnicity, education, partnered status, body mass index (BMI), and time since surgery were included in models. Data were analyzed using t-tests and ordinal logistic regression modeling with significance set at $P<0.05$. Results: Women $(\mathrm{n}=118)$ compared to men $(\mathrm{n}=168)$ were less likely to be partnered $(25 \%$ vs $55 \%, P<0.0001)$. There were no differences for age, ethnicity, education, BMI, or time since surgery. Regression modeling for fatigue showed women to have lower HR-QOL compared to men $(0.65$ decrease, $P<0.01)$, adjusted for time since surgery (modest positive association, $P<0.05$ ). Regression modeling for sleep disruption also showed women to have poorer HR-QOL (0.57 decrease, $P<0.01$ ), adjusted for age (modest positive association, $P<0.001$ ). CRC surgical controls without ostomy showed no significant gender difference for either HR-QOL item. Qualitative narrative comments suggest sleep disruption is associated with fear of, or actual, leakage during hours of sleep. Conclusions: Women CRC survivors with ostomies report more fatigue and sleep disruption that may contribute to poorer HR-QOL compared to their male counterparts. Higher rates of fatigue for women are consistent with gender differences in other health conditions. These findings can provide a foundation for gender-specific ostomy interventions to improve sleep quality.

\section{Abstract PS1-25 \\ Statin Use is Associated With a Reduced Risk of Colon Cancer Recurrence}

Jody M. Jackson, RN, BSN, HealthPartners Research Foundation; Daniel M. Anderson, MD, MPH, Regions Hospital Cancer Center; Amy L. Butani, BA, HealthPartners Research Foundation; Stephen E. Asche, MA, HealthPartners Research Foundation; Cheri J. Rolnick, MPH, PhD, HealthPartners Research Foundation

Background: A significant body of data suggests that statin use reduces the risk of developing colon and other cancers, although other data have not confirmed these findings. Aims: To examine whether statin use helps prevent recurrence of colon cancer in patients with resected disease. Methods: We conducted a retrospective analysis of patients treated for stage I-III colon cancer in a large metropolitan health system. Incident cases of colon cancer between 1995, and the present were identified through the tumor registry of the health plan-owned hospital. All identified cases were then crosschecked against the HMO membership database. Data regarding statin use was pulled from the HMO outpatient pharmacy database. For each patient all available data from the 5-year window prior to first diagnosis of cancer until either recurrence or most recent data available was obtained. Demographic data and survival data from the tumor registry were then combined with pharmacy data regarding statin use in a single database for analysis. Recurrence and time to recurrent disease were compared in statin users and non-users. Results: A total of 358 colon cancer cases were available for analysis. Median follow-up of patients after diagnosis of colon cancer was 49 months. Eighty-nine patients $(24.9 \%)$ used statin drugs for some period of time with a median of 780 days of use. Median age of all patients enrolled was 69 years (range, 24-95 yrs). Statin use was higher in patients ages $61-75$ years $(33 \%)$ than in those aged $24-60$ years $(22 \%)$ or those aged $76-95$ years $(19 \%)$ $P=0.02$ ). There was no association between statin use and stage at diagnosis. Only 6 of 89 patients $(6.7 \%)$ with any statin use relapsed as opposed to 43 of $269(16 \%)$ of patients with no statin use (Fisher's exact, $P=0.03$ ). Kaplan-Meier analysis of relapse curves found a significant difference in relapse between groups with a 2-year recurrence rate of $3.7 \%$ among statin users and $10.1 \%$ among non-users ( $\log$ Rank, $P=0.03$, using age stratification). Conclusions: Statin use was associated with a decreased risk of recurrence in patients diagnosed with stages I-III colon cancer in this study. Further study of statin use in the prevention of colon cancer is warranted.

\section{Abstract PS1-26 \\ The Content of Physician-Patient Discussions During Routine Health Maintenance Exams}

Jennifer Elston Lafata, PhD, Henry Ford Health System; Greg Cooper, MD, University Hospitals of Cleveland, and Case Comprehensive Cancer Center; George Divine, PhD, Department of Biostatistics and Research Epidemiology, Henry Ford Health System; Susan Flocke, PhD, Departments of Family Medicine and Epidemiology and Biostatistics, Case Western Reserve University; Laura Siminoff, $\mathrm{PhD}$, Department of Behavioral Sciences and Health Promotion, Virginia Commonwealth University School of Public Health; Kurt Stange, MD, Departments of Family Medicine and Epidemiology and Biostatistics, Case Western Reserve University; Tracy Wunderlich, MA, Center for Health Services Research, Henry Ford Health System

Background: Little is known about the content of routine health maintenance exams (HMEs) delivered by primary care physicians (PCP) in today's busy primary care practices. We describe the frequency of patient-physician cancer screening discussions amidst the competing demands of other preventive services during routine HMEs to understand HME content in the real world of primary care. Methods: We use direct observation of PCP-patient interactions during HMEs to examine discussions around breast, colorectal (CRC), cervical, lung and prostate cancer screening and other lifestyle topics. Visits represent the first 103 visits completed in an NIH-funded study using in-office observations, audio-recordings and patient surveys to study CRC screening recommendations. Physician participants $(n=34)$ are general internists or family physicians practicing in a large integrated delivery system in southeast Michigan with patient-specific electronic medical record prompts for evidence-based cancer screening. Patient participants were aged 50-80 years who scheduled an HME with a participating physician and were due for CRC screening (i.e., no colonoscopy or barium enema within 10 years, flexible sigmoidoscopy within 5 years, or fecal occult blood testing (FOBT) within 12 months). Results: CRC screening was mentioned in $96 \%$ of observed HMEs. Among visits by women $(n=74), 85 \%$ mentioned breast and $51 \%$ cervical cancer screening. Among visits by men $(n=29), 72 \%$ mentioned prostate cancer screening. Lung cancer screening was mentioned in $15 \%$ of visits. The physician initiated these discussions more than $80 \%$ of the time, with the exception of lung cancer screening (53\%). Colonoscopy was overwhelmingly the procedure recommended for CRC screening $(82 \%)$, followed by FOBT (37\%) and others (4\%). Other topics frequently discussed were exercise $(88 \%)$, cholesterol $(81 \%)$, smoking $(68 \%)$, weight $(68 \%)$, blood pressure $(65 \%)$, and diet $(65 \%)$. While these discussions were usually initiated by physicians, patients were relatively more likely to initiate discussions around weight (41\%) and diet (35\%). Conclusions: In this study 